\title{
Hyperfine induced electron spin and entanglement dynamics in double quantum dots: The case of separate baths
}

\author{
B. Erbe and J. Schliemann \\ Institut für Theoretische Physik, Universität Regensburg, D-93053 Regensburg, Germany
}

(Received 25 January 2012; published 16 April 2012)

\begin{abstract}
We consider a system of two strongly coupled electron spins in zero magnetic field, each of which is interacting with an individual bath of nuclear spins via the hyperfine interaction. Applying the long-spin approximation that we introduced in a previous paper [Europhys. Lett. 95, 47009 (2011)] (here each bath is replaced by a single long spin), we numerically study the electron spin and entanglement dynamics. We demonstrate that the decoherence time is scaling with the bath size according to a power law. As expected, the decaying part of the dynamics decreases with increasing bath polarization. However, surprisingly it turns out that, under certain circumstances, combining quantum dots of different geometry to the double dot setup has a very similar effect on the magnitude of the spin decay. Finally, we show that even for a comparatively weak exchange coupling the electron spins can be fully entangled.
\end{abstract}

DOI: 10.1103/PhysRevB.85.155127

PACS number(s): 76.20.+q, 76.60.Es, 85.35.Be

\section{INTRODUCTION}

The Loss-DiVincenco proposal is one of the most promising concepts for solid-state quantum information processing. Here, electron spins confined in semiconductor quantum dots are utilized as qubits. ${ }^{1,2}$ The central drawback of this approach is the fast decoherence caused by the coupling of the electron spin qubits to the nuclear spins of the host material via the hyperfine interaction. ${ }^{3-10}$ For related reviews, the reader is referred to Refs. 11-15. Other nanostructures in which similar situations arise are given by carbon nanotube quantum dots, ${ }^{16}$ phosphorus donors in silicon, ${ }^{17}$ and nitrogen vacancies in diamond. ${ }^{18-20}$

However, the hyperfine interaction allows us to access the nuclear spins efficiently. Hence, when it comes to utilizing them instead of the electron spins for quantum information purposes, vice turns into virtue and the hyperfine interaction gets a very advantageous character. Examples in this context are given by the possibility to build up an interface between light and nuclear spins, ${ }^{21,22}$ to polarize nuclear spin baths, ${ }^{23-25}$ to set up long-lived quantum ${ }^{26,27}$ and classical $^{28}$ memory devices, or to generate entanglement. ${ }^{29}$

Following the idea to take advantage of the hyperfine interaction, in a recent paper $^{30}$ we investigated a system of two exchange-coupled electron spins, each of which is interacting with an individual bath of nuclear spins via the hyperfine interaction. In contrast to most of the approaches considered in the context of hyperfine interaction, ${ }^{31-36}$ no magnetic field, enabling for a perturbative treatment of the problem, was applied to the electron spins. Using exact diagonalization studies, we demonstrated that the nuclear baths can be swapped and fully entangled, provided they are large enough. In order to be able to numerically consider the required system sizes, we introduced the so-called long-spin approximation (LSA). Here, we assumed homogeneous couplings within each of the baths and considered them to be highly polarized. This allows us to replace them by two single long spins. Interestingly, the spectrum of the two-bath model with homogeneous couplings, studied in a preceding publication, ${ }^{37}$ exhibits systematically degenerate multiplets under certain conditions. Motivated by this, we distinguished between systems with and without inversion symmetry, i.e., a formal exchange of the central as well as the bath spins. In the latter case, quantum dots of different geometry are combined to a double dot setup. Surprisingly, it turned out that here the swap performance is much better.

In the present paper, we apply the LSA in order to study the electron-spin dynamics. The results complement those of Refs. 30 and 37 and, in particular, those of Ref. 38, where we studied the electron spin evolution assuming the electrons to interact with a common bath of nuclear spins via homogeneous couplings.

The paper is organized as follows: In Sec. II we introduce the model and the methods. In particular, we discuss in detail the applicability of the LSA with respect to the electron-spin dynamics. We then study the spin and entanglement dynamics in the limit of an exchange coupling, which is much larger than the hyperfine energy scale. Here, the nuclear baths act as a perturbation. This is a particularly interesting case, as exceptionally long decoherence times can be expected. In Sec. III we focus on the time evolution of the electron spins. In a first step we study basic dynamical properties. In particular we demonstrate that in certain parameter ranges the process of decoherence is incomplete. Furthermore, we find a simple empirical rule describing the dynamical signatures of different initial states. We then quantitatively investigate the decoherence time and the magnitude of the spin decay. As expected from Ref. 38, the decoherence time scales with the system size according to a power law. As already mentioned, in Ref. 30 it was demonstrated that the nuclear-spin dynamics strongly benefit from combining quantum dots of different geometry to the double-dot setup. In full generality, this result can be confirmed only in certain parameter regimes. In Sec. IV we then focus on the entanglement dynamics and demonstrate that, surprisingly, even for a comparatively weak exchange coupling, the electron spins can be fully entangled.

\section{MODEL AND METHODS}

The hyperfine interaction in a double quantum dot is described by the Hamiltonian

$$
H=\vec{S}_{1} \cdot \sum_{i=1}^{N} A_{i}^{(1)} \vec{I}_{i}+\vec{S}_{2} \cdot \sum_{i=1}^{N} A_{i}^{(2)} \vec{I}_{i}+J_{\mathrm{ex}} \vec{S}_{1} \cdot \vec{S}_{2}
$$


where $\vec{S}_{i}$ are the electron and $\vec{I}_{i}$ are the nuclear spins. The parameter $J_{\mathrm{ex}}$ denotes an exchange coupling between the two electron spins, which can be adjusted in a range of $\left[-10^{-3}, 10^{-3}\right] \mathrm{eV}$. The constants $A_{i}^{(1)}, A_{i}^{(2)}$ are the hyperfine couplings of the two electron spins. In a realistic quantum dot, these are proportional to the electronic wave function of the $j$ th electron at the site $\vec{r}_{i}$ of the $i$ th nuclear spin:

$$
A_{i}^{(j)} \propto\left|\Psi^{(j)}\left(\vec{r}_{i}\right)\right|^{2} .
$$

For typical GaAs quantum dots, this leads to an interaction with $N \sim 10^{6}$ nuclear spins and the overall hyperfine coupling strength of the $j$ th electron,

$$
A^{(j)}:=\sum_{i=1}^{N} A_{i}^{(j)},
$$

is of the order of $\left[10^{-4}, 10^{-5}\right] \mathrm{eV}$ (see Ref. 39).

Due to the spatial variation of the electronic wave function, the hyperfine couplings are clearly spatially dependent. However, for any set of hyperfine coupling constants, the Hamiltonian, obviously, conserves the total spin $\vec{J}=\vec{S}_{1}+$ $\vec{S}_{2}+\sum_{i=1}^{N} \vec{I}_{i}$. This is a very helpful symmetry for exact numerical diagonalizations of the Hamiltonian matrix, ${ }^{3,39}$ through which we will gain the dynamics of the system in what follows. Here we consider the eigensystem of the Hamiltonian

$$
H\left|\psi_{i}\right\rangle=E_{i}\left|\psi_{i}\right\rangle
$$

and decompose the initial state $|\alpha\rangle$ into a sum of energy eigenstates:

$$
|\alpha\rangle=\sum_{i} \alpha_{i}\left|\psi_{i}\right\rangle
$$

Applying the time evolution operator $U=e^{-\frac{i}{\hbar} H t}$ and tracing out the nuclear degrees of freedom then gives the reduced density matrix for the electrons,

$$
\begin{aligned}
\rho_{e}(t) & =\operatorname{Tr}_{n}(|\alpha(t)\rangle\langle\alpha(t)|) \\
& =\sum_{i, j} \alpha_{i} \alpha_{j}^{*} e^{-\frac{i}{\hbar}\left(E_{i}-E_{j}\right) t} \operatorname{Tr}_{n}\left(\left|\psi_{i}\right\rangle\left\langle\psi_{j}\right|\right),
\end{aligned}
$$

from which the dynamics of all observables can be calculated. For further details see Ref. 38.

In Ref. 38 we investigated the case of two electron spins coupled to a common nuclear spin bath. In what follows, however, we consider the case of two separate baths as depicted schematically in Fig. 1. In the first case, the two electron spins are assumed to be very close to each other so that both interact with the same group of nuclear spins, whereas in the present

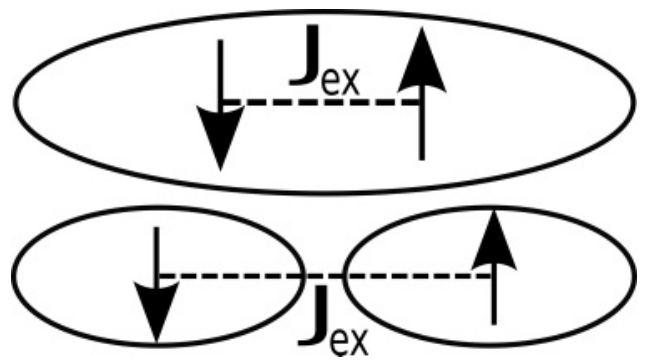

FIG. 1. Illustration of the one-bath and the two-bath model case they are spatially more separated, leading to an interaction with an individual group. The realistic situation of a double quantum dot will, of course, lie between these two extreme cases.

As already mentioned, in the present paper we apply the LSA to the two-bath system. In the following subsections we give a detailed discussion of the model with a particular focus on its limitations.

\section{A. The long spin approximation}

Let us consider two separate spin baths of equal size with homogeneous couplings to one of the two electron spins each and introduce $\vec{I}_{j}=\sum_{i=1}^{N_{j}} \vec{I}_{i j}$, where the $\vec{I}_{i j}$ are the $N_{j}$ nuclear spins the $j$ th electron spin interacts with. This means that $N=N_{1}+N_{2}$ and $\sum_{i=1}^{N} \vec{I}_{i}=\vec{I}_{1}+\vec{I}_{2}$, where, for simplicity, we will consider $N_{1}=N_{2}$ in what follows. Now the squares of the total spin of each bath are separate conserved quantities. Moreover, the same holds for the square of any sum over a subset of spins of each bath,

$$
\left[H, \vec{I}_{j}^{2}\right]=\left[H,\left\{\vec{K}_{j}^{2}\right\}\right]=0,
$$

where we have, for the sake of brevity, denoted the set of all the latter operators of the $j$ th bath as $\left\{\vec{K}_{j}^{2}\right\}$. The corresponding quantum numbers $\left\{K_{j}\right\}$ can be used to characterize specific Clebsch-Gordan decompositions of each bath.

The initial state $|\alpha\rangle$ is given by a direct product between the initial state of the electron spins $\left|\alpha_{e}\right\rangle$ and the initial state of the baths $\left|\alpha_{n}\right\rangle$. Provided the two dots are spatially well-separated, the two resulting baths have to be considered as practically uncorrelated. Hence, the state of the nuclear baths is again a direct product between the states of the two baths, $\left|\beta_{j}\right\rangle$. In general, such a state reads

$$
\left|\beta_{j}\right\rangle=\sum_{I_{j}, m_{j},\left\{K_{j}\right\}} \beta_{j}^{I_{j}, m_{j},\left\{K_{j}\right\}}\left|I_{j}, m_{j},\left\{K_{j}\right\}\right\rangle,
$$

where $\left|I_{j}, m_{j},\left\{K_{j}\right\}\right\rangle$ are the eigenstates of $\vec{I}_{j}^{2}$. If the respective bath is now strongly polarized, the number of contributing multiplets in Eq. (8) drastically decreases. ${ }^{38}$ If we are close to full positive or negative polarization, we can drop the quantum numbers $\left\{K_{j}\right\}$ and consider the initial state to be given by $\left|I, m_{j}\right\rangle$ with $m_{j} \approx \pm I$. Due to Eq. (7), no "cross terms" between different multiplets contribute to the dynamics and all physics is then captured in the LSA Hamiltonian

$$
H_{L S A}=\frac{A^{(1)}}{2 I} \vec{S}_{1} \cdot \vec{I}_{1}+\frac{A^{(2)}}{2 I} \vec{S}_{2} \cdot \vec{I}_{2}+J_{e x} \vec{S}_{1} \cdot \vec{S}_{2},
$$

sketched in Fig. 2. The coupling constants $A^{(j)} / 2 I$ result directly from Eq. (3) by considering $I_{i j}=1 / 2$ : As all couplings $A_{i}^{(j)}$ are chosen to be equal to each other, Eq. (3) yields $A_{i}^{(j)}=A^{(j)} / N_{j}$. The quantum number $I_{j}$ ranges from 0 to $N_{j} / 2$. As our model is based on highly polarized baths, we choose the maximal value. Together with $N_{1}=N_{2}$, this yields $A_{i}^{(j)}=A^{(j)} / 2 I$. Although the LSA Hamiltonian is not exactly solvable, the approximation of the baths by single long spins reduces the dimension of the problem so that exact numerical diagonalizations are possible on arbitrary subspaces even for comparatively large baths. 


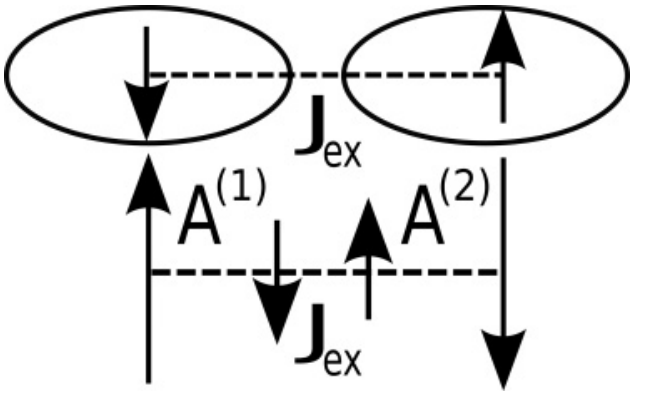

FIG. 2. The two baths are approximated by two long spins.

Accounting for the $J^{z}$ symmetry, the nuclear state $\left|\alpha_{n}\right\rangle$ explicitly reads

$$
\left|\alpha_{n}\right\rangle=|I, M-m-i\rangle|I, i\rangle .
$$

Here, $M$ denotes the quantum number associated with $J^{z}$ and $m$ the one related to $S^{z}$. The parameter $i$ is introduced in order to account for the deviations from $m_{j}= \pm I$. Hence, it has to be chosen in the vicinity of $I$ or $(M-m-I)$, respectively. Note that for an initial state, which is a simple product state like Eq. (10), all dynamics is caused by the flip-flop terms

$$
\frac{1}{2}\left(S_{i}^{+} S_{j}^{-}+S_{i}^{-} S_{j}^{+}\right)=\vec{S}_{i} \cdot \vec{S}_{j}-S_{i}^{3} S_{j}^{3}
$$

in $H$ or $H_{L S A}$, respectively. This is exactly the part of the Hamiltonian that is eliminated in most of the approaches by applying a strong magnetic field to the central spin system (see Refs. 31-36). In Refs. 30 and 38 we also concentrated on the dynamics that are purely due to the flip-flop terms.

\section{B. Homogeneous couplings on long time scales}

In Ref. 38 we considered the one-bath model illustrated in the upper panel of Fig. 1 for homogeneous couplings and initial states with a very low bath polarization $p_{b}:=(N-$ $\left.2 N_{D}\right) / N$ of $(1 / N)$. Here, $N_{D}$ denotes the number of flipped spins in the bath. The central spin dynamics shows periodic behavior. This clearly has to be regarded as an artifact caused by the homogeneity of the couplings. For short time scales, meaning times much smaller than the recurrence time, the results for decoherence times found there compare well with experimental values.

As explained above, within the LSA we assume the couplings to be homogeneous and the baths to be highly polarized. However, high polarizations naturally lead to long time scales for the electron spin decoherence times. Consequently, it has to be analyzed to what extent the two assumptions of the LSA contradict each other. In Ref. 30 we already investigated this question with respect to the nuclear spin dynamics, where we considered a Gaudin model, as corresponding to one of the first terms in Eq. (1). We found that, qualitatively, inhomogeneities become less important with increasing polarization. Typically, in such a context, one would give a quantitative argument by evaluating the fidelity (to be precisely defined below) rather than studying the dynamics on a qualitative level. However, with respect to the nuclear spin dynamics considered in Ref. 30 this does not make sense, obviously, as the bath consists of many spins so that a certain value of $\left\langle I^{z}\right\rangle$ can be realized by a whole set of nuclear states.

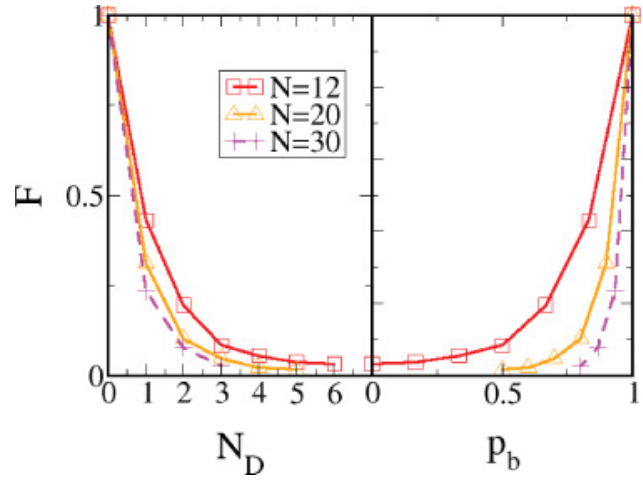

FIG. 3. (Color online) The time-averaged fidelity is plotted against the number of flipped spins in the bath (left panel) and against the bath magnetization (right panel). We consider a randomly correlated initial bath state with coefficients in $[-1,1]$ for $N=$ $12,20,30$. The fidelity strongly increases with increasing polarization. Furthermore, it decreases with an increasing number of bath spins, where the decrease gets weaker as the baths become larger.

In the following, we again consider a usual Gaudin model and investigate the time-averaged fidelity $F$ with respect to homogeneous and inhomogeneous couplings via exact diagonalization. This is given by

$$
F=\frac{1}{T} \int_{t=0}^{T} d t\left|\left\langle U_{\mathrm{h}} \alpha \mid U_{\mathrm{ih}} \alpha\right\rangle\right|,
$$

with $U_{\mathrm{h}}$ and $U_{\mathrm{ih}}$ being the time evolution operators for the homogeneous or inhomogeneous Hamiltonian, respectively. We choose an initial state that is a direct product between an electron spin pointing upward and a randomly correlated bath state. This is a superposition of all possible states with (in our case) real random coefficients, which we choose in the interval $[-1,1]$. Randomly correlated states lead to highly reproducible results and can, therefore, be regarded as generic. ${ }^{3,39}$

The results are shown in Fig. 3. We fix three different system sizes of $N=12,20,30$ bath spins for a reasonably long period $T=400(\hbar / A)$. In the left panel, we plot $F$ against $N_{D}$. In order to get a better comparison between the different system sizes, in the right panel we show the same data plotted against the bath polarization $p_{b}$. Obviously, the fidelity is strongly increasing with the bath polarization. Furthermore, it decreases with an increasing number of bath spins. Note that the different curves approach each other with increasing number of bath spins.

The highest experimentally feasible polarizations are around $80 \%$, as reported in Ref. 40. On first sight, the results shown in Fig. 3 indicate that even for such high polarizations, considering homogeneous couplings on comparatively long time scales is restricted to extremely small systems. This would strongly contradict the purpose of the LSA. However, it turns out that the fidelity is an extremely sensitive measure underestimating the applicability of the LSA: In Fig. 4 we plot the spin dynamics for inhomogeneous and homogeneous couplings for $N_{D}=4 \Leftrightarrow p_{b}=(1 / 3)$, corresponding to a very low fidelity of $F=0.055194$. Such a small value clearly suggests that the dynamics in the inhomogeneous and the homogeneous case are fundamentally different. The amplitude of $\left\langle S_{z}(t)\right\rangle$ decaying to zero without any recurrence on the 


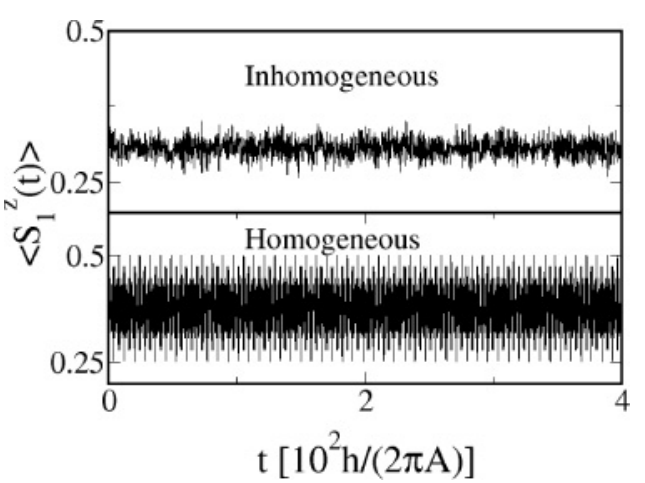

FIG. 4. Central spin dynamics for inhomogeneous and homogeneous couplings for $N=12$ and $N_{D}=4 \Leftrightarrow p_{b}=(1 / 3)$, as corresponding to $F=0.055194$. Just as described in the legend of Fig. 3, we consider a randomly correlated initial bath state with coefficients in $[-1,1]$. In both cases the spin is oscillating around a very similar mean value, where the amplitude is significantly larger for the case of homogeneous couplings. Hence, even in this extreme case of a very small fidelity, the dynamics are still somewhat similar to each other.

considered time scales would be an example of a natural expectation for the first case (compare, e.g., the results presented in Refs. 3 and 39 with those of Refs. 38 and 41). Furthermore, one would guess that the time-averaged values of $\left\langle S_{z}(t)\right\rangle$ in the inhomogeneous and the homogeneous case strongly differ from each other. However, as can been seen from Fig. 4, neither of these expectations are met. This means that even very small fidelities correspond to a rather good qualitative agreement of the dynamics. Considering highly polarized baths, as done within the LSA, it is therefore justified to choose homogeneous couplings even on comparatively long time scales.

\section{ELECTRON SPIN DYNAMICS}

In the following, we restrict ourselves to the limit $J_{\text {ex }} /(A / 2 I) \gg 1$, where we defined $A=A^{(1)}+A^{(2)}$. Here, the dynamics is dominated by the electron-spin coupling term and the baths act as a perturbation. As a consequence, long decoherence times, enabling us, e.g., to fully entangle the two electron spins, have to be expected. We will distinguish between a "strong coupling" and an "ultrastrong coupling" limit. In the first case an only moderately large exchange coupling, $J_{\mathrm{ex}} / A \approx 1$, is considered so that the condition $J_{\mathrm{ex}} /(A / 2 I) \gg 1$ is realized mainly through the length of the bath spins, whereas in the second case we choose a very strong exchange coupling, meaning that here we already have $J_{\text {ex }} / A \gg 1$. As $I_{1}=I_{2}$, a zero "detuning" $\Delta:=A^{(2)}-A^{(1)}$ is associated with a system invariant under inversions $1 \leftrightarrow 2 .^{37}$ Physically, a detuning different from zero corresponds to dots of different geometry combined to a double quantum dot. Throughout the paper we will consider the dynamics on subspaces with fixed magnetization. It therefore suffices to investigate the $z$ components of the spins. Furthermore, due to their strong coupling, the dynamics of the two electron spins can be read off from each other even for $\Delta \neq 0$. Therefore, we always focus on the time evolution of the first electron spin. Note that the energy scale is given by $(A / 2 I)$ and consequently the time will be given in units of $h I / \pi A$.

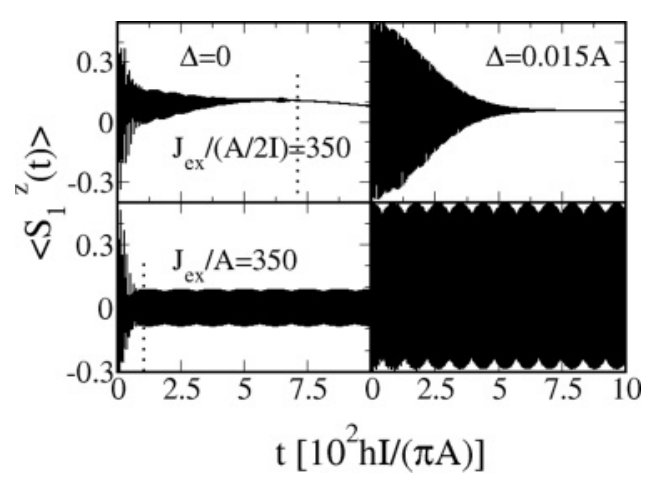

FIG. 5. Electron-spin dynamics for the strong and the ultrastrong coupling case $J_{\mathrm{ex}} /(A / 2 I), J_{\mathrm{ex}} / A=350$, with $\Delta=0$ in the left column and $\Delta \neq 0$ in the right one. We consider $I=120$ and $M_{r}=0.17$. The initial state is given by $|\alpha\rangle=|\Uparrow \downarrow\rangle|M-I, I\rangle$. As illustrated by the dotted lines, we choose the point at which the amplitude does not change anymore as the decoherence time. We find very regular dynamics, where the decoherence times are obviously larger in the case of broken inversion symmetry $\Delta \neq 0$. Furthermore, the oscillations do not fully decay in the ultrastrong coupling case.

\section{A. Basic dynamical properties}

In order to give a basic impression of the dynamics, in Figs. 5 and 6 we fix $J_{\text {ex }} /(A / 2 I)=350$ for the strong and $J_{\mathrm{ex}} / A=350$ for the ultrastrong coupling case and plot the dynamics of the first electron spin for $I=120$. We consider the relatively low "magnetization" $M_{r}:=M /(2 I+1)=0.17$. This means that we concentrate on initially nearly antiparallel baths. We study the inversion invariant case $\Delta=0$ as well as $\Delta \neq 0$. All initial states considered in Figs. 5 and 6 have an antiparallel electron spin configuration $\left|\alpha_{e}\right\rangle=|\Uparrow \Downarrow\rangle$. In Fig. 5, we consider an initial nuclear state with a maximally negative $z$ component of the first bath spin, $\vec{I}_{1}$. This corresponds to $i=I$ in Eq. (10). We clearly see that in the ultrastrong coupling limit, the time evolution for initial states of the above-mentioned form does not fully decay. Indeed, for small, inversion invariant systems this is also the case in the strong coupling limit. Varying $i$ slightly away from $I$, the dynamics in the ultrastrong coupling case does not show any qualitative

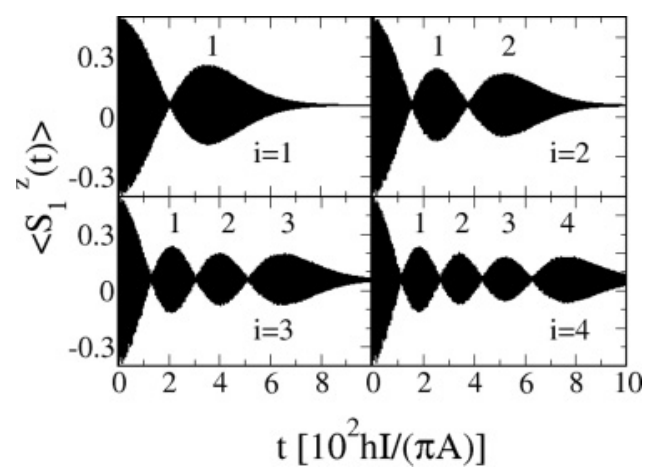

FIG. 6. Electron spin dynamics for the strong coupling case $J_{\text {ex }} /(A / 2 I)=350$ and $\Delta=0.015$. We consider $I=120$ and $M_{r}=$ 0.17 . The initial states are given by $|\alpha\rangle=|\Uparrow \Downarrow\rangle|M-I+i, I-i\rangle$ with $i=1,2,3,4$. We find dynamics with an envelope, decaying in a quite similar way to the one for $i=0$ shown in Fig. 5. However, here additional beatings occur. Their number is equal to $i$. 
change. As can be seen in Fig. 6, this is also the case for the envelope of the dynamics in the strong coupling limit. However, here additional beatings occur. Surprisingly, there is a clear empirical rule concerning these additional low-frequency oscillations: If the $z$ component of the first bath spin deviates by $i$ from the maximal negative value, $\left|\alpha_{n}\right\rangle=$ $|M-I+i, I-i\rangle$, the dynamics shows exactly $i$ beatings. In Fig. 6, the case of broken inversion symmetry is considered, where the beatings are particularly pronounced. At present, we are not able to explain this effect. However, it seems that nontrivial dynamical regularities are typical for central spin models with homogeneous couplings. Indeed, in Ref. 38 we reported on a rule for the one bath model, which relates the number of flipped spins in the initial state of the bath to the number of local extrema in the oscillations of the central spins. Also, the dynamics has been calculated on a fully analytical level, and we have not been able to give an explanation of these regularities.

\section{B. Decoherence time and magnitude of the spin decay}

In direct analogy to the investigations in Ref. 38, in the following we investigate the scaling of the decoherence time with the spin length. It is clear that such an investigation can not yield perfectly reliable values, as the spin length is, of course, restricted to comparatively small values due to the limited computational power. Consider, for example, $M_{r}=0$. Here, the dimension of the Hilbert space is given by $(8 I+2)$, limiting the length of the spins to values of the order of $I \sim 10^{2}$. Still, the results give a clear idea about the type of scaling and allow for a qualitative comparison between different parameter regimes. In the following we concentrate on initial states $|\alpha\rangle=|\Uparrow \downarrow\rangle|M-I, I\rangle$ for $M_{r}=0.17$. As already explained, $i$ has to be in the vicinity of $I$ and the envelope remains unaffected when varying $i$ slightly away from its maximal value. Hence, the results for $|\alpha\rangle=|\Uparrow \downarrow\rangle|M-I, I\rangle$ can be regarded as generic. As can be seen from Fig. 5, in the ultrastrong coupling limit $\left\langle S_{1}^{z}(t)\right\rangle$ does not decay to a constant value, but oscillations of quite regular shape remain; i.e., the decoherence process is not complete. Therefore, we define the time from which on the amplitude does not change anymore than the decoherence time. Numerically, this is realized by dividing the time axis in intervals with a length larger than the period of the regular oscillations and determining the maximal value in each interval. If this value does not change anymore over a fixed number of intervals, the lower bound of the first interval in which the respective value appeared is chosen as the decoherence time. In the left panels of Fig. 5, this choice is illustrated by the dotted lines.

In Ref. 38, it has been shown for the one-bath model that the decoherence time scales with the size of the bath according to a power law $\sim N^{-v}$. Indeed, we find the same behavior for the present case. In Figs. 7 and 8, the decoherence times for the strong and the ultrastrong coupling case are plotted against the spin length $I$ on a double logarithmic scale. For the inversion symmetric case we consider $J_{\mathrm{ex}} /(A / 2 I), J_{\mathrm{ex}} / A=$ 350 and $J_{\mathrm{ex}} /(A / 2 I)=175$, where it obviously does not make any sense to choose a second value for the ultrastrong coupling limit. For the case of broken inversion symmetry we fix $J_{\text {ex }} /(A / 2 I), J_{\text {ex }} / A=350$ and fix $\Delta=0.015 A, 0.019 A$ for the

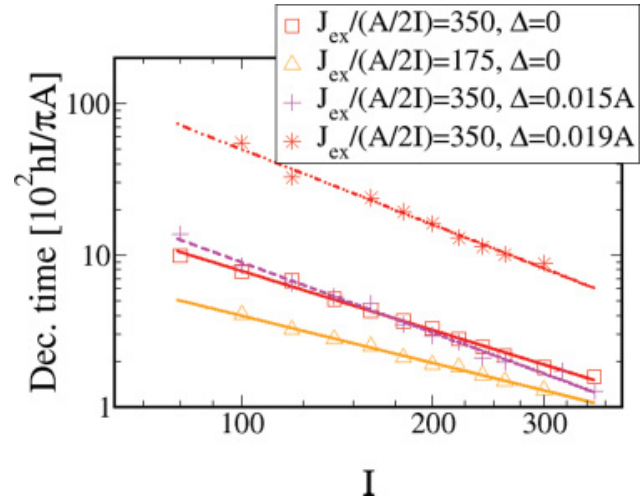

FIG. 7. (Color online) Scaling of the decoherence time with the bath spin length $I$ on a double logarithmic scale for the strong-coupling case. Different coupling ratios $J_{\mathrm{ex}} /(A / 2 I)$ and detunings $\Delta$ are considered. We choose the initial state $|\alpha\rangle=$ $|\Uparrow \Downarrow\rangle|M-I, I\rangle$ for $M_{r}=0.17$. We find power laws $\sim I^{-v}$ with $v=$ $1.29\left[J_{\mathrm{ex}} /(A / 2 I)=350, \Delta=0\right], v=1.03\left[J_{\mathrm{ex}} /(A / 2 I)=175, \Delta=\right.$ $0], \quad v=1.54\left[J_{\mathrm{ex}} /(A / 2 I)=350, \Delta=0.015 A\right], \quad$ and $\quad v=1.65$ $\left[J_{\mathrm{ex}} /(A / 2 I)=350, \Delta=0.019 A\right]$. Although the limit $J_{\mathrm{ex}} /(A / 2 I) \gg$ 1 is left unaltered, surprisingly the scaling changes with the coupling ratio. Breaking the inversion symmetry leads to an increase of the parameter $v$.

strong coupling and $\Delta=0.001 A, 0.006 A$ for the ultrastrong coupling case. The values for the latter are chosen to be particularly small, because, as exemplified in the bottom panels of Fig. 5, the dynamics is highly sensitive with respect to a change of the detuning and become completely coherent on any relevant time scale for larger values.

As expected, for the strong-coupling case, the decoherence time is scaling much stronger than for the ultrastrong coupling limit. Note that the values for the ultrastrong case are much smaller only due to the fact that here the dynamics does not fully decay. As can be seen from Fig. 7, in the strong-coupling

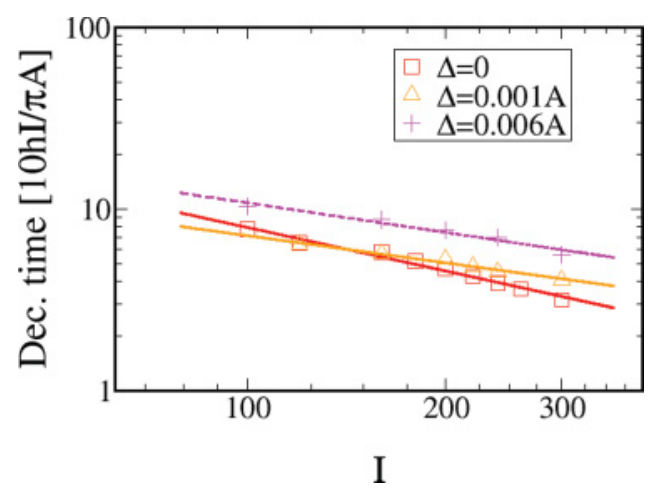

FIG. 8. (Color online) Scaling of the decoherence time with the bath spin length $I$ on a double logarithmic scale for the ultrastrong coupling case. We fix $J_{\text {ex }} / A=350$ and vary $\Delta$ weakly away from zero. We choose the initial state $|\alpha\rangle=|\Uparrow \Downarrow\rangle|M-I, I\rangle$ for $M_{r}=0.17$. The curves are again fitted to power laws $\sim I^{-v}$ with $v=0.79(\Delta=$ $0), v=0.49(\Delta=0.001 A)$, and $v=0.54(\Delta=0.006 A)$. Note that the absolute of the decoherence times are smaller than in the strong coupling case because the dynamics does not fully decay. Breaking the inversion symmetry leads to a decrease of $v$ to $v \approx 0.5$, which is the value found in Ref. 38 for the one-bath model. 
limit the scaling does change significantly with the coupling ratio $J_{\mathrm{ex}} /(A / 2 I)$. This is surprising as a small change in the ratio leaves the limit $J_{\text {ex }} /(A / 2 I) \gg 1$ unaltered and, hence, one would expect the scaling to be insensitive against a change of the coupling ratio. Furthermore, the absolute values of the decoherence time clearly decrease with decreasing coupling ratio $J_{\text {ex }} /(A / 2 I)$ as expected. However, as a counterintuitive effect, the scaling with the system size turns out to be weaker for the smaller of the two ratios. Breaking the inversion symmetry has a significant effect in the strong as well as the ultrastrong coupling limit. In the first case, the exponent $v$ increases, whereas in the latter it decreases to $v \approx 0.5$. This is the value derived in Ref. 38 for the one-bath model.

As explained in the preceding subsection, in the ultrastrong coupling case the dynamics does not show full decoherence. If the spin length $I$ is small and we have $\Delta=0$, this is also the case for only strongly coupled electron spins. We now analyze the scaling of the decaying part of the dynamics as a function of the magnetization and, in the ultrastrong coupling case, as a function of the detuning $\Delta$. Concerning the strong coupling case the results are, obviously, only of fundamental interest. Even in SiGe and carbon-based quantum dots (only around $4.7 \%$ of the $\mathrm{Si}$ isotopes are spin carrying, ${ }^{42}$ and in carbon only $1 \%{ }^{43}$ ), the electron spins interact with a few thousands of nuclear spins.

Note that our LSA model is valid only for relatively small and relatively large magnetizations $M_{r}$, which corresponds to nuclear spin baths highly polarized in either the same or opposite directions. However, in Fig. 9 we plot the amplitude, defined as the difference between a local maximum and the following local minimum, for the whole range of $M_{r}$, which corresponds to either parallel or antiparallel nuclear spin baths.

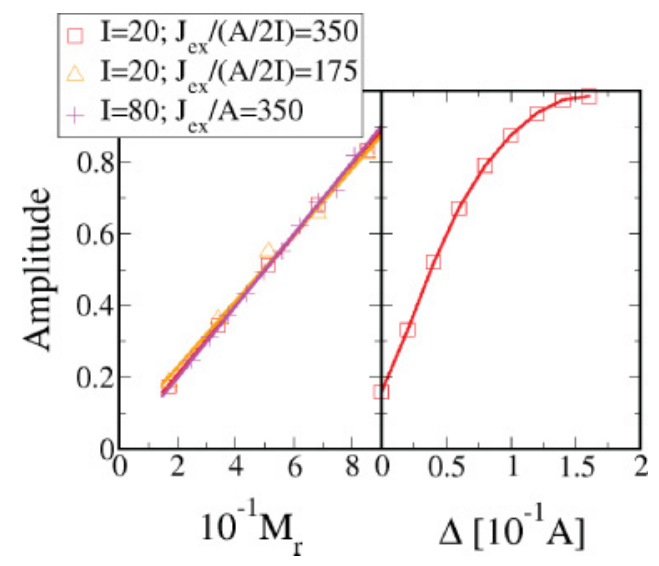

FIG. 9. (Color online) Scaling of the amplitude, given by the difference of a local maximum and the following local minimum, with the magnetization and the detuning for the cases, where the dynamics does not fully decay. In the left panel we fix $\Delta=0$. For the strong-coupling case we consider $I=20$ and $J_{\text {ex }} /(A / 2 I)=350,175$. For the ultrastrong coupling case we fix $I=80$ and $J_{\text {ex }} / A=350$. We choose the initial state $|\alpha\rangle=|\Uparrow \downarrow\rangle|M-I, I\rangle$. In all cases we find a linear dependence of the amplitude on the magnetization with gradients $a$ close to one. The values are given $a=0.97$ $\left[J_{\mathrm{ex}} /(A / 2 I)=350\right], a=0.92\left[J_{\mathrm{ex}} /(A / 2 I)=175\right]$, and $a=1.0$ $\left(J_{\mathrm{ex}} / A=350\right)$. In the right panel we plot the the amplitude against the detuning for $M_{r} \approx 0.17$. Here we find a highly nonlinear dependence.
We consider the strong as well as the ultrastrong coupling case. For the first one we fix $I=20$ and two values for the coupling ratio $J_{\text {ex }} /(A / 2 I)=350,175$. In both cases we find a linear dependence with a gradient close to one, meaning that the ratio does not significantly influence the decaying part. Concerning the ultrastrong coupling case, we set $I=80$ and consider $J_{\mathrm{ex}} / A=350$. The scaling is practically identical to the one for the strong coupling case. As already discussed in the preceding section, we found that for the ultrastrong coupling case the decaying part is not only influenced by the magnetization but also by the detuning. In the right panel of Fig. 9, we plot the amplitude against the detuning for a fixed magnetization $M_{r}=0.17$. In contrast to a variation of the magnetization, here we find a highly nonlinear dependence, which can not be fitted by some simple power law.

In Ref. 30 we demonstrated that a nonzero detuning is very advantageous with respect to swapping and entangling the nuclear spin baths. When it comes to the electron spin dynamics, however, in general this is the case only the ultrastrong coupling limit.

\section{ENTANGLEMENT DYNAMICS}

We now close the discussion of the electron-spin dynamics with an investigation of the entanglement between the two electron spins. In order to quantify the nonclassical correlations, we consider the concurrence defined by ${ }^{44}$

$$
C(t)=\max \left\{0, \sqrt{\lambda_{1}}-\sqrt{\lambda_{2}}-\sqrt{\lambda_{3}}-\sqrt{\lambda_{4}}\right\},
$$

where $\lambda_{i}$ are the eigenvalues of the non-Hermitian matrix $\rho_{e}(t) \tilde{\rho}_{e}(t)$ in decreasing order. Here, $\tilde{\rho}_{e}(t)$ is given by $\left(\sigma_{y} \otimes\right.$ $\left.\sigma_{y}\right) \rho_{e}^{*}(t)\left(\sigma_{y} \otimes \sigma_{y}\right)$, where $\rho_{e}^{*}(t)$ denotes the complex conjugate of $\rho_{e}(t)$ - the reduced density matrix of the electrons as defined in Eq. (6).

In the following, we ask to what extent it is possible to entangle initially uncorrelated electron spins. Therefore, we again consider initial states with electron spin configurations $|\Uparrow \Downarrow\rangle$. In particular, we are interested in a lower bound for the ratio $J_{\text {ex }} /(A / 2 I)$, meaning that we adjust the couplings $J_{\text {ex }}, A_{1}, A_{2}$ to the lowest possible ratio so that the concurrence still becomes equal to one. As to be expected, the lower bound lies in the ultrastrong coupling limit. However, surprisingly it turns out that it is not determined by the ratio $J_{\mathrm{ex}} / A$, but only by $J_{\text {ex }} / \max \left\{A_{1}, A_{2}\right\}$. The concrete value of this ratio depends on the initial state of the nuclear spins. An upper bound is given by the (as explained above, unphysical) case of randomly correlated states. As an empirical rule of thumb, here, we find

$$
\frac{J_{\mathrm{ex}}}{\max \left\{A_{1}, A_{2}\right\}} \geqslant 8.8 .
$$

In Fig. 10 we illustrate the rule by plotting the dynamics for randomly correlated initial states with coefficients in $[-1,1]$ by considering parameters satisfying and violating Eq. (14). We choose a rather small system of $I=40$ and concentrate on the case of broken inversion symmetry $\Delta=0.019 A$. We plot the time evolution for a low polarization of $M_{r}=0.09$ in the left panel and fix a rather high polarization of $M_{r}=0.86$ 


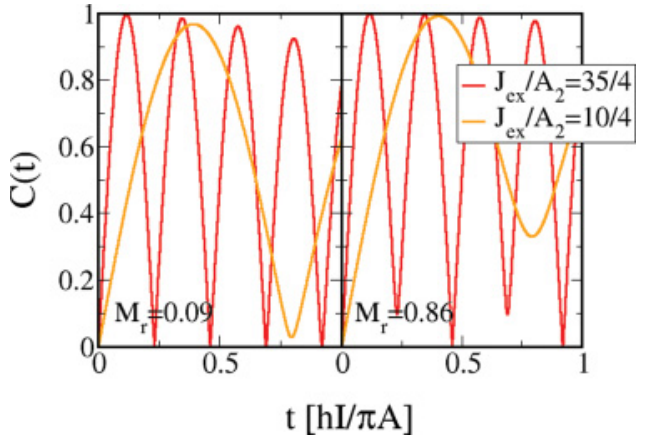

FIG. 10. (Color online) Time evolution of the concurrence between the two central spins for $I=40$. We consider the case of a low polarization $\left(M_{r}=0.09\right)$ in the left panel and the case of a high polarization $\left(M_{r}=0.86\right)$ in the right panel. In both cases we choose a detuning of $\Delta=0.019 A$ and consider $J_{\text {ex }} / A_{2}=8.8$, which satisfies Eq. (14), and $J_{\mathrm{ex}} / A_{2}=2.5$, which violates Eq. (14). The nuclear spins are in a randomly correlated state with coefficients in $[-1,1]$ initially. One clearly sees that if Eq. (14) is satisfied, the concurrence becomes one, whereas for a stronger coupling to the baths, the electron spins cannot be fully entangled.

in the right panel. It is visible that the maximal value of the entanglement drops slightly under one if Eq. (14) is violated.

\section{CONCLUSION}

In summary, we numerically studied the electron spin and entanglement dynamics in a system of two strongly coupled electron spins, each of which is interacting with an individual bath of nuclear spins via the hyperfine interaction. We applied the LSA introduced in Ref. 30, where the two baths are replaced by two single long spins, and focused on the limit of an exchange coupling much larger than the hyperfine energy scale. Here we distinguished between a strong and an ultrastrong coupling case. We demonstrated that the decoherence time scales with the size of the baths according to a power law. As expected, it turned out that the decaying part decreases with increasing polarization. However, surprisingly it also decreases with increasing detuning, provided the electrons are bound ultrastrongly. Hence, with respect to the electron-spin dynamics, the advantageous character of a nonzero detuning, found in Ref. 30 for the time evolution of the nuclear baths, can only be confirmed in the ultrastrong coupling limit. Finally, we demonstrated that it is possible to fully entangle the electron spins even for a comparatively weak exchange coupling.

\section{ACKNOWLEDGMENT}

This work was supported by DFG via SFB 631.
${ }^{1}$ D. Loss and D. P. DiVincenzo, Phys. Rev. A 57, 120 (1998).

${ }^{2}$ R. Hanson, L.P. Kouwenhoven, J. R. Petta, S. Tarucha, and L. M. K. Vandersypen, Rev. Mod. Phys. 79, 1217 (2007).

${ }^{3}$ A. V. Khaetskii, D. Loss, and L. Glazman, Phys. Rev. Lett. 88, 186802 (2002).

${ }^{4}$ A. V. Khaetskii, D. Loss, and L. Glazman, Phys. Rev. B 67, 195329 (2003).

${ }^{5}$ A. C. Johnson, J. R. Petta, J. M. Taylor, A. Yacoby, M. D. Lukin, C. M. Marcus, M. P. Hanson, and A. C. Gossard, Nature 435, 925 (2005).

${ }^{6}$ F. H. L. Koppens, J. A. Folk, J. M. Elzerman, R. Hanson, L. H. Willems van Beveren, I. T. Vink, H. P. Tranitz, W. Wegscheider, L. P. Kouwenhoven, and L. M. K. Vandersypen, Science 309, 1346 (2005).

${ }^{7}$ J. R. Petta, A. C. Johnson, J. M. Taylor, E. A. Laird, A. Yacoby, M. D. Lukin, C. M. Marcus, M. P. Hanson, and A. C. Gossard, Science 309, 2180 (2005).

${ }^{8}$ F. H. L. Koppens, C. Buizert, K. J. Tielrooij, I. T. Vink, K. C. Nowack, T. Meunier, L. P. Kouwenhoven, and L. M. K. Vandersypen, Nature 442, 766 (2006).

${ }^{9}$ F. H. L. Koppens, K. C. Nowack, and L. M. K. Vandersypen, Phys. Rev. Lett. 100, 236802 (2008).

${ }^{10}$ P. F. Braun, X. Marie, L. Lombez, B. Urbaszek, T. Amand, P. Renucci, V. K. Kalevick, K. V. Kavokin, O. Krebs, P. Voisin, and Y. Masumoto, Phys. Rev. Lett. 94, 116601 (2005).

${ }^{11}$ J. Schliemann, A. V. Khaetskii, and D. Loss, J. Phys.: Condens. Matter 15, R1809 (2003).

${ }^{12}$ W. Zhang, N. Konstantinidis, K. A. Al-Hassanieh, and V. V. Dobrovitski, J. Phys.: Condens. Matter. 19, 083202 (2007).

${ }^{13}$ D. Klauser, D. V. Bulaev, W. A. Coish, and D. Loss, e-print arXiv:0706.1514.
${ }^{14}$ W. A. Coish and J. Baugh, Phys. Status Solidi B 246, 2203 (2009).

${ }^{15}$ J. M. Taylor, J. R. Petta, A. C. Johnson, A. Yacoby, C. M. Marcus, and M. D. Lukin, Phys. Rev. B 76, 035315 (2007).

${ }^{16}$ H. O. H. Churchill, A. J. Bestwick, J. W. Harlow, F. Kuemmeth, D. Marcos, C. H. Stwertka, S. K. Watson, and C. M. Marcus, Nature Physics 5, 321 (2009).

${ }^{17}$ E. Abe, K. M. Itoh, J. Isoya, and S. Yamasaki, Phys. Rev. B 70, 033204 (2004).

${ }^{18}$ F. Jelezko, T. Gaebel, I. Popa, A. Gruber, and J. Wrachtrup, Phys. Rev. Lett. 92, 076401 (2004).

${ }^{19}$ L. Childress, M. V. G. Dutt, J. M. Taylor, A. S. Zibrov, F. Jelezko, J. Wrachtrup, P. R. Hemmer, and M. D. Lukin, Science 314, 281 (2006).

${ }^{20}$ R. Hanson, V. V. Dobrovitski, A. E. Feiguin, O. Gywat, and D. D. Awschalom, Science 320, 352 (2008).

${ }^{21}$ H. Schwager, J. I. Cirac, and G. Giedke, Phys. Rev. B 81, 045309 (2010).

${ }^{22}$ H. Schwager, J. I. Cirac, and G. Giedke, New. J. Phys. 12, 043026 (2010).

${ }^{23}$ J. M. Taylor, A. Imamoglu, and M. D. Lukin, Phys. Rev. Lett. 91, 246802 (2003)

${ }^{24}$ H. Christ, J. I. Cirac, and G. Giedke, Solid State Sci. 11, 965 (2009).

${ }^{25}$ H. Christ, J. I. Cirac, and G. Giedke, Phys. Rev. B 75, 155324 (2007).

${ }^{26}$ J. M. Taylor, C. M. Marcus, and M. D. Lukin, Phys. Rev. Lett. 90, 206803 (2003)

${ }^{27}$ J. J. L. Morton, A. M. Tyryshkin, R. M. Brown, S. Shankar, B. W. Lovett, A. Ardavan, T. Schenkel, E. E. Haller, J. W. Ager, and S. A. Lyon, Nature (London) 455, 1085 (2008).

${ }^{28}$ G. Austing, C. Payette, G. Yu, and J. Gupta, Jpn. J. Appl. Phys. 48, 04C143 (2009). 
${ }^{29}$ H. Christ, J. I. Cirac, and G. Giedke, Phys. Rev. B 78, 125314 (2008).

${ }^{30}$ B. Erbe and J. Schliemann, Europhys. Lett. 95, 47009 (2011).

${ }^{31}$ A. V. Khaetskii, D. Loss, and L. Glazman, Phys. Rev. Lett. 88, 186802 (2002).

${ }^{32}$ A. V. Khaetskii, D. Loss, and L. Glazman, Phys. Rev. B 67, 195329 (2003).

${ }^{33}$ W. A. Coish and D. Loss, Phys. Rev. B 70, 195340 (2004).

${ }^{34}$ W. A. Coish and D. Loss, Phys. Rev. B 72, 125337 (2005).

${ }^{35}$ D. Klauser, W. A. Coish, and D. Loss, Phys. Rev. B 73, 205302 (2006).

${ }^{36}$ D. Klauser, W. A. Coish, and D. Loss, Phys. Rev. B 78, 205301 (2008).
${ }^{37}$ B. Erbe and J. Schliemann, J. Phys. A: Math. Theor. 43, 492002 (2010).

${ }^{38}$ B. Erbe and J. Schliemann, Phys. Rev. B 81, 235324 (2010).

${ }^{39}$ J. Schliemann, A. V. Khaetskii, and D. Loss, Phys. Rev. B 66, 245303 (2002).

${ }^{40}$ P. Maletinsky, Ph.D. thesis, ETH Zuerich (2008).

${ }^{41}$ M. Bortz and J. Stolze, J. Stat. Mech. (2007) P06018.

${ }^{42}$ A. Wild, J. Sailer, J. Ntzel, G. Abstreiter, S. Ludwig, and D. Bougeard, New J. Phys. 12, 113019 (2010).

${ }^{43}$ J. Fischer, B. Trauzettel, and D. Loss, Phys. Rev. B 80, 155401 (2009).

${ }^{44}$ W. K. Wootters, Phys. Rev. Lett. 80, 2245 (1998). 